\title{
Applications of first order differential subordination for functions with positive real part
}

\author{
Om P. Ahuja, Sushil Kumar and V Ravichandran
}

\begin{abstract}
Several inclusions between the class of functions with positive real part and the class of starlike univalent functions associated with lemniscate of Bernoulli are obtained by making use of the well-known theory of differential subordination. Further, these inclusions give sufficient conditions for normalized analytic functions to belong to some subclasses of starlike functions. The results also provide sharp version of some previously known results.
\end{abstract}

Mathematics Subject Classification (2010): 30C45.

Keywords: Differential subordination, starlike function, lemniscate of Bernoulli, functions with positive real part.

\section{Introduction}

Let $\mathcal{A}$ denote the class of analytic functions $f$ on the disk $\mathbb{D}:=\{z \in \mathbb{C}:|z|<1\}$ and normalized by the condition $f(0)=0=f^{\prime}(0)-1$. Let $\mathcal{S}$ be the subset of $\mathcal{A}$ of univalent functions. An analytic function $f$ defined on $\mathbb{D}$ is subordinate to the analytic function $g$ on $\mathbb{D}$ (or $g$ is superordinate to $f$ ), if there exists an analytic function $w: \mathbb{D} \rightarrow \mathbb{D}$, with $w(0)=0$, such that $f=g \circ w$. Furthermore, if $g$ is univalent in $\mathbb{D}$, then $f \prec g$ is equivalent to $f(0)=g(0)$ and $f(\mathbb{D}) \subseteq g(\mathbb{D})$, see $[15]$. Let $p$ be an analytic function in $\mathbb{D}$ with positive real part and $p(0)=1$. The function $p(z)=(1+z) /(1-z)$ is a leading example of the function with positive real part such that $p(\mathbb{D})$ is the right-half plane. Goluzin [7] discussed the first order differential subordination $z p^{\prime}(z) \prec z q^{\prime}(z)$ and proved that, whenever $z q^{\prime}(z)$ is convex, the subordination $p(z) \prec q(z)$ holds and the function $q$ is the best dominant. After this basic result, many authors established several generalizations of first order differential subordination. The general theory of differential subordination is discussed in the monograph by Miller and Mocanu [14]. In 1989, Nunokawa et al. [16] proved that if subordination $1+z p^{\prime}(z) \prec 1+z$ holds, then subordination $p(z) \prec 1+z$ also holds. 
In 2007, Ali et al. [2] extended this result and determined the estimates on $\beta$ for which the subordination $1+\beta z p^{\prime}(z) / p^{j}(z) \prec(1+D z) /(1+E z)(j=0,1,2)$ implies the subordination $p(z) \prec(1+A z) /(1+B z)$, where $A, B, D, E \in[-1,1]$. In 2013, Omar and Halim [17] determined the condition on $\beta$ in terms of complex number $D$ and real $E$ with $-1<E<1$ and $|D| \leq 1$ such that $1+\beta z p^{\prime}(z) / p^{j}(z) \prec(1+D z) /(1+E z)$ $(j=0,1,2)$ implies $p(z) \prec \sqrt{1+z}$. These results are not sharp. Recently, Kumar and Ravichandran [11] obtained sharp estimates on $\beta$ for which the subordination $1+\beta z p^{\prime}(z) / p^{j}(z)(j=0,1,2)$ is subordinate to $\sqrt{1+z},(1+A z) /(1+B z)$ and some another functions with positive real part whenever the subordination $p(z) \prec e^{z},(1+$ $A z) /(1+B z)$ holds. They further used these results to determine some sufficient conditions for the function $f \in \mathcal{A}$ to be in certain well-known subclasses of starlike functions. For more details, see [3, 5, 6, 20, 23, 25].

Motivated by all this work, we determine the sharp bound on $\beta$ so that $p(z) \prec \mathcal{P}(z)$ where $\mathcal{P}(z)$ is a function with positive real part like $\sqrt{1+z},(1+A z) /(1+B z), e^{z}$, $\varphi_{s}(z), \varphi_{q}(z), \varphi_{0}(z)$ and $\varphi_{C}(z)$, where $\varphi_{0}(z):=1+\frac{z}{k}((k+z) /(k-z))(k=\sqrt{2}+1)$, $\varphi_{s}(z):=1+\sin z, \varphi_{c}(z):=1+\frac{4}{3} z+\frac{2}{3} z^{2}$ and $\varphi_{q}(z):=z+\sqrt{1+z^{2}}$, whenever $1+\beta z p^{\prime}(z) / p^{j}(z) \prec \sqrt{1+z},(j=0,1,2)$. Many of our subordination results in this paper improve the corresponding non-sharp results obtained by earlier authors in $[1,9,13]$.

\section{Subordination results}

Our first result gives a bound on $\beta$ so that $1+\beta z p^{\prime}(z) \prec \sqrt{1+z}$ implies that the function $p$ is subordinate to several well-known starlike functions.

Theorem 2.1. Let the function $p$ be analytic in $\mathbb{D}, p(0)=1$ and $1+\beta z p^{\prime}(z) \prec \sqrt{1+z}$. Then the following subordination results hold:

(a) If $\beta \geq \frac{2(\sqrt{2}-1+\log 2-\log (1+\sqrt{2}))}{\sqrt{2}-1} \approx 1.09116$, then $p(z) \prec \sqrt{1+z}$.

(b) If $\beta \geq \frac{2(1-\log 2)}{3-2 \sqrt{2}} \approx 3.57694$, then $p(z) \prec \varphi_{0}(z)$.

(c) If $\beta \geq \frac{2(1-\log 2)}{\sin (1)} \approx 0.729325$, then $p(z) \prec \varphi_{s}(z)$.

(d) If $\beta \geq(2+\sqrt{2})(1-\log 2) \approx 1.044766$, then $p(z) \prec \varphi_{q}(z)$.

(e) If $\beta \geq 3(1-\log 2) \approx 0.920558$, then $p(z) \prec \varphi_{c}(z)$.

(f) Let $-1<B<A<1$ and $B_{0}=\frac{2-\log 4-\sqrt{2}+\log (1+\sqrt{2})}{\sqrt{2}-\log (1+\sqrt{2}+1)} \approx 0.151764$.

If either

(i) $B<B_{0}$ and $\beta \geq \frac{2(1-B)(1-\log 2)}{A-B} \approx 0.613706 \frac{1-B}{A-B}$

or

(ii) $B>B_{0}$ and $\beta \geq \frac{2(1+B)(\sqrt{2}-1+\log 2-\log (1+\sqrt{2}))}{A-B} \approx 0.451974 \frac{1+B}{A-B}$, then $p(z) \prec(1+A z) /(1+B z)$.

The bounds on $\beta$ are sharp.

The following lemma will be used in our investigations.

Lemma 2.2. [15, Theorem 3.4h, p. 132] Let $q$ be analytic in $\mathbb{D}$ and let $\psi$ and $\nu$ be analytic in a domain $U$ containing $q(\mathbb{D})$ with $\psi(w) \neq 0$ when $w \in q(\mathbb{D})$. 
Set $Q(z):=z q^{\prime}(z) \psi(q(z))$ and $h(z):=\nu(q(z))+Q(z)$. Suppose that (i) either $h$ is convex, or $Q$ is starlike univalent in $\mathbb{D}$ and $(i i) \operatorname{Re}\left(z h^{\prime}(z) / Q(z)\right)>0$ for $z \in \mathbb{D}$. If $p$ is analytic in $\mathbb{D}$, with $p(0)=q(0), p(\mathbb{D}) \subseteq U$ and

$$
\nu(p(z))+z p^{\prime}(z) \psi(p(z)) \prec \nu(q(z))+z q^{\prime}(z) \psi(q(z)),
$$

then $p(z) \prec q(z)$, and $q$ is best dominant.

Proof of Theorem 2.1. The function $q_{\beta}: \overline{\mathbb{D}} \rightarrow \mathbb{C}$ defined by

$$
q_{\beta}(z)=1+\frac{2}{\beta}(\sqrt{1+z}-\log (1+\sqrt{1+z})+\log 2-1) .
$$

is analytic and is a solution of the differential equation $1+\beta z q_{\beta}^{\prime}(z)=\sqrt{1+z}$. Consider the functions $\nu(w)=1$ and $\psi(w)=\beta$. The function $Q: \overline{\mathbb{D}} \rightarrow \mathbb{C}$ is defined by

$$
Q(z)=z q_{\beta}^{\prime}(z) \psi\left(q_{\beta}(z)\right)=\beta z q_{\beta}^{\prime}(z) .
$$

Since $\sqrt{1+z}-1$ is starlike function in $\mathbb{D}$, it follows that function $Q$ is starlike. Also note that the function $h(z)=\nu\left(q_{\beta}(z)\right)+Q(z)$ satisfies $\operatorname{Re}\left(z h^{\prime}(z) / Q(z)\right)>0$ for $z \in \mathbb{D}$. Therefore, by making use of Lemma 2.2, it follows that

$$
1+\beta z p^{\prime}(z) \prec 1+\beta z q_{\beta}^{\prime}(z) \text { implies } p(z) \prec q_{\beta}(z) \text {. }
$$

Each of the conclusion in (a)-(f) is $p(z) \prec \mathcal{P}(z)$ for appropriate $\mathcal{P}$ and this holds if the subordination $q_{\beta}(z) \prec \mathcal{P}(z)$ holds.

If $q_{\beta}(z) \prec \mathcal{P}(z)$, then $\mathcal{P}(-1)<q_{\beta}(-1)<q_{\beta}(1)<\mathcal{P}(1)$. This gives a necessary condition for $p \prec \mathcal{P}$ to hold. Surprisingly, this necessary condition is also sufficient. This can be seen by looking at the graph of the respective functions.

(a) On taking $\mathcal{P}(z)=\sqrt{1+z}$, the inequalities $q_{\beta}(-1) \geq 0$ and $q_{\beta}(1) \leq \sqrt{2}$ reduce to $\beta \geq \beta_{1}$ and $\beta \geq \beta_{2}$, where

$$
\beta_{1}=2(1-\log 2) \text { and } \beta_{2}=2(\sqrt{2}-1+\log 2-\log (1+\sqrt{2})) /(\sqrt{2}-1)
$$

respectively. Therefore, the subordination $q_{\beta}(z) \prec \sqrt{1+z}$ holds only if

$$
\beta \geq \max \left\{\beta_{1}, \beta_{2}\right\}=\beta_{2} .
$$

(b) Consider $\mathcal{P}(z)=\varphi_{0}(z)$. A simple calculation shows that the inequalities $q_{\beta}(-1) \geq \varphi_{0}(-1)$ and $q_{\beta}(1) \leq \varphi_{0}(1)$ reduce to $\beta \geq \beta_{1}$ and $\beta \geq \beta_{2}$, where

$$
\beta_{1}=2(1-\log 2) /(3-2 \sqrt{2}) \text { and } \beta_{2}=2(\sqrt{2}-1+\log 2-\log (1+\sqrt{2}))
$$

respectively. Thus the subordination $q_{\beta}(z) \prec \varphi_{0}(z)$ holds only if

$$
\beta \geq \max \left\{\beta_{1}, \beta_{2}\right\}=\beta_{1} .
$$

(c) Consider $\mathcal{P}(z)=\varphi_{s}(z)$. The inequalities $q_{\beta}(-1) \geq \varphi_{s}(-1)$ and $q_{\beta}(1) \leq \varphi_{s}(1)$ reduce to $\beta \geq \beta_{1}$ and $\beta \geq \beta_{2}$, where

$$
\beta_{1}=\frac{2(1-\log 2)}{\sin (1)} \text { and } \beta_{2}=\frac{2(\sqrt{2}-1+\log 2-\log (1+\sqrt{2}))}{\sin (1)}
$$

respectively. The subordination $q_{\beta}(z) \prec \varphi_{s}(z)$ holds if $\beta \geq \max \left\{\beta_{1}, \beta_{2}\right\}=\beta_{1}$. 
(d) Consider $\mathcal{P}(z)=\varphi_{q}(z)$. The inequalities $q_{\beta}(-1) \geq \varphi_{q}(-1)$ and $q_{\beta}(1) \leq \varphi_{q}(1)$ give $\beta \geq \beta_{1}$ and $\beta \geq \beta_{2}$, where

$$
\beta_{1}=(2+\sqrt{2})(1-\log 2) \text { and } \beta_{2}=\sqrt{2}(\sqrt{2}-1+\log 2-\log (1+\sqrt{2}))
$$

respectively. The subordination $q_{\beta}(z) \prec \varphi_{q}(z)$ holds if $\beta \geq \max \left\{\beta_{1}, \beta_{2}\right\}=\beta_{1}$.

(e) Consider $\mathcal{P}(z)=\varphi_{c}(z)$. From the inequalities

$$
\varphi_{c}(-1) \leq q_{\beta}(-1) \text { and } q_{\beta}(1) \leq \varphi_{c}(1),
$$

we get

$$
\beta \geq 3(1-\log 2) \text { and } \beta \geq 2(\sqrt{2}-1+\log 2-\log (1+\sqrt{2}))
$$

respectively. Thus the subordination $q_{\beta}(z) \prec \varphi_{c}(z)$ holds if

$$
\beta \geq \max \{3(1-\log 2), 2(\sqrt{2}-1+\log 2-\log (1+\sqrt{2}))\}=3(1-\log 2) .
$$

(f) Consider $\mathcal{P}(z)=(1+A z) /(1+B z)$. From the inequalities

$$
q_{\beta}(-1) \geq(1-A) /(1-B) \text { and } q_{\beta}(1) \leq(1+A) /(1+B),
$$

we note that $\beta \geq \beta_{1}$ and $\beta \geq \beta_{2}$, where

$$
\beta_{1}=\frac{2(1-B)(1-\log 2)}{A-B} \text { and } \beta_{2}=\frac{2(1+B)(\sqrt{2}-1+\log 2-\log (1+\sqrt{2}))}{A-B}
$$

respectively. A simple calculation gives

$$
\beta_{1}-\beta_{2}=2(1-\log 2)+(1+B)(\log (1+\sqrt{2})-\sqrt{2}) .
$$

We note that $\beta_{1}-\beta_{2} \geq 0$ if $B<B_{0}$ and $\beta_{1}-\beta_{2} \leq 0$ if $B>B_{0}$ where

$$
B_{0}=\frac{2-\log 4-\sqrt{2}+\log (1+\sqrt{2})}{\sqrt{2}-\log (1+\sqrt{2}+1)} .
$$

The necessary subordination $p(z) \prec(1+A z) /(1+B z)$ holds if $\beta \geq \max \left\{\beta_{1}, \beta_{2}\right\}$.

Remark 2.3. The subordination results in part (a) and (f) in Theorem 2.1 were also investigated by the authors in [1, Lemma 2.1, p. 1019] and [9, Lemma 2.1, p. 3], but their results were non-sharp.

In 1985, Padmanabhan and Parvatham [18] introduced a unified classes of starlike and convex functions using convolution with the function of the form $z /(1-z)^{\alpha}$, $\alpha \in \mathbb{R}$. Later, Shanmugam [21] considered the class $\mathcal{S}_{g}^{*}(h)$ of all $f \in \mathcal{A}$ satisfying $z(f * g)^{\prime} /(f * g) \prec h$ where $h$ is a convex function, $g$ is a fixed function in $\mathcal{A}$.

Denote by $\mathcal{S}^{*}(h)$ and $\mathcal{K}(h)$, the subclass $\mathcal{S}_{g}^{*}(h)$, when $g$ is $z /(1-z)$ and $z /(1-z)^{2}$ respectively. In 1992, Ma and Minda [12] considered a weaker assumption that $h$ is a function with positive real part whose range is symmetric with respect to real axis and starlike with respect to $h(0)=1$ with $h^{\prime}(0)>0$ and proved distortion, growth, and covering theorems. The class $\mathcal{S}^{*}(h)$ generalizes many subclasses of $\mathcal{A}$, for example, $\mathcal{S}^{*}[A, B]:=\mathcal{S}^{*}((1+A z) /(1+B z))(-1 \leq B<A \leq 1)[8], \mathcal{S}_{e}^{*}:=\mathcal{S}^{*}\left(e^{z}\right)[13]$, $\mathcal{S}_{s}^{*}:=\mathcal{S}^{*}\left(\varphi_{s}(z)\right)[4], \mathcal{S}_{C}^{*}:=\mathcal{S}^{*}\left(\varphi_{c}(z)\right)[22], \mathcal{S}_{R}^{*}:=\mathcal{S}^{*}\left(\varphi_{0}(z)\right)[10]$, and $\mathcal{S}_{q}^{*}:=\mathcal{S}^{*}\left(\varphi_{q}(z)\right)$ [19]. The function $\sqrt{1+z}$ is associated with the class $\mathcal{S}_{L}^{*}:=\mathcal{S}^{*}(\sqrt{1+z})$ [24], introduced by Sokół and Stankiewicz. This class consists of the function $f \in \mathcal{A}$ such that $w(z):=z f^{\prime}(z) / f(z)$ lies in the region bounded by the right half of the lemniscate of 
Bernoulli given by $\left|w^{2}-1\right|<1$. The lemniscate of Bernoulli is a best known plane curve resembling the symbol $\infty$. It was named after James Bernoulli who considered it in elasticity theory in 1694. In geometry, the lemniscate is a plane curve defined by two given points $F_{1}$ and $F_{2}$, known as foci, at distance $2 a$ from each other as the locus of points $\mathrm{P}$ so that $P F_{1} \cdot P F_{2}=a^{2}$. The equation of lemniscate may be written as $\left(x^{2}+y^{2}\right)^{2}=2 a^{2}\left(x^{2}-y^{2}\right)$. The lemniscate in the complex plane is the locus of $z=x+i y$ such that $\left|z^{2}-a^{2}\right|=a^{2}$.

Remark 2.4. Let the function $f \in \mathcal{A}$ satisfying the following subordination

$$
1+\beta \frac{z f^{\prime}(z)}{f(z)}\left(1-\frac{z f^{\prime}(z)}{f(z)}+\frac{z f^{\prime \prime}(z)}{f^{\prime}(z)}\right) \prec \sqrt{1+z} .
$$

Then the following are sufficient conditions for $f$ to be in various subclasses of $\mathcal{S}$.

(a) If $\beta \geq \frac{2(\sqrt{2}-1+\log 2-\log (1+\sqrt{2}))}{\sqrt{2}-1} \approx 1.09116$, then $f \in \mathcal{S}_{L}^{*}$.

(b) If $\beta \geq \frac{2(1-\log 2)}{3-2 \sqrt{2}} \approx 3.57694$, then $f \in \mathcal{S}_{R}^{*}$.

(c) If $\beta \geq \frac{2(1-\log 2)}{\sin (1)} \approx 0.729325$, then $f \in \mathcal{S}_{s}^{*}$.

(d) If $\beta \geq(2+\sqrt{2})(1-\log 2) \approx 1.044766$, then $f \in \mathcal{S}_{q}^{*}$.

(e) If $\beta \geq 3(1-\log 2) \approx 0.920558$, then $f \in \mathcal{S}_{c}^{*}$.

(f) Let $-1<B<A<1$ and $B_{0}=\frac{2-\log 4-\sqrt{2}+\log (1+\sqrt{2})}{\sqrt{2}-\log (1+\sqrt{2}+1)} \approx 0.151764$.

If either $B<B_{0}$ and $\beta \geq \frac{2(1-B)(1-\log 2)}{A-B} \approx 0.613706 \frac{1-B}{A-B}$ or $B>B_{0}$ and

$$
\beta \geq \frac{2(1+B)(\sqrt{2}-1+\log 2-\log (1+\sqrt{2}))}{A-B} \approx 0.451974 \frac{1+B}{A-B},
$$

then $f \in \mathcal{S}^{*}[A, B]$.

The bounds on $\beta$ are sharp.

Next result provides a bound on $\beta$ so that $1+\beta z p^{\prime}(z) / p(z) \prec \sqrt{1+z}$ implies $p$ is subordinate to some well-known starlike functions.

Theorem 2.5. Let the function $p$ be analytic in $\mathbb{D}, p(0)=1$ and

$$
1+\beta z p^{\prime}(z) / p(z) \prec \sqrt{1+z} .
$$

Then the following subordination results hold:

(a) If $\left.\beta \geq \frac{2(\log 2-1)}{\log (2 \sqrt{2}-2}\right) \approx 3.26047$, then $p(z) \prec \varphi_{0}(z)$.

(b) If $\beta \geq \frac{2(\sqrt{2}-1+\log (2)-\log (\sqrt{2}+1))}{\log (1+\sin (1))} \approx 0.740256$, then $p(z) \prec \varphi_{s}(z)$.

(c) If $\beta \geq \frac{2(\log 2-1)}{\log (\sqrt{2}-1)} \approx 0.696306$, then $p(z) \prec \varphi_{q}(z)$.

(d) If $\beta \geq 2(1-\log 2) \approx 0.613706$, then $p(z) \prec e^{z}$.

(e) If $-1<B<A<1$ and $\beta \geq \max \left\{\beta_{1}, \beta_{2}\right\}$ where

$$
\begin{aligned}
& \beta_{1}=\frac{2(1-\log 2)}{\log (1-B)-\log (1-A)} \text { and } \beta_{2}=\frac{2(\sqrt{2}-1+\log 2-\log (1+\sqrt{2}))}{\log (1+A)-\log (1+B)}, \\
& \text { then } p(z) \prec(1+A z) /(1+B z) .
\end{aligned}
$$

The bounds on $\beta$ are best possible. 
Proof. The function $q_{\beta}: \overline{\mathbb{D}} \rightarrow \mathbb{C}$ defined by

$$
q_{\beta}(z)=\exp \left(\frac{2}{\beta}(\sqrt{1+z}-\log (1+\sqrt{1+z})+\log 2-1)\right)
$$

is analytic and is a solution of the differential equation

$$
1+\beta z q_{\beta}^{\prime}(z) / q_{\beta}(z)=\sqrt{1+z} .
$$

Define the functions $\nu(w)=1$ and $\psi(w)=\beta / w$.

The function $Q: \overline{\mathbb{D}} \rightarrow \mathbb{C}$ defined by

$$
Q(z):=z q_{\beta}^{\prime}(z) \psi\left(q_{\beta}(z)\right)=\beta z q_{\beta}^{\prime}(z) / q_{\beta}(z)=\sqrt{1+z}-1
$$

is starlike in $\mathbb{D}$. The function $h(z):=\nu\left(q_{\beta}(z)\right)+Q(z)=1+Q(z)$ satisfies $\operatorname{Re}\left(z h^{\prime}(z) / Q(z)\right)>0$ for $z \in \mathbb{D}$. Therefore, by using Lemma 2.2 , we see that the subordination

$$
1+\beta \frac{z p^{\prime}(z)}{p(z)} \prec 1+\beta \frac{z q_{\beta}^{\prime}(z)}{q_{\beta}(z)}
$$

implies $p(z) \prec q_{\beta}(z)$. As the similar lines of the proof of Theorem 2.1, the proofs of parts (a)-(e) are completed.

Remark 2.6. The subordination in part (d) and (e) of Theorem 2.5 were earlier investigated in [13, Theorem 2.16(c), p. 10] and [9, Lemma 2.3, p. 5] where non-sharp results were obtained.

Remark 2.7. Let the function $f \in \mathcal{A}$ satisfies the following subordination

$$
1+\beta\left(1-\frac{z f^{\prime}(z)}{f(z)}+\frac{z f^{\prime \prime}(z)}{f^{\prime}(z)}\right) \prec \sqrt{1+z} .
$$

Then the following are sufficient conditions for $f$ to be in various subclasses of $\mathcal{S}$.

(a) If $\left.\beta \geq \frac{2(\log 2-1)}{\log (2 \sqrt{2}-2}\right) \approx 3.26047$, then $f \in \mathcal{S}_{R}^{*}$.

(b) If $\beta \geq \frac{2(\sqrt{2}-1+\log (2)-\log (\sqrt{2}+1))}{\log (1+\sin (1))} \approx 0.740256$, then $f \in \mathcal{S}_{s}^{*}$.

(c) If $\beta \geq \frac{2(\log 2-1)}{\log (\sqrt{2}-1)} \approx 0.696306$, then $f \in \mathcal{S}_{q}^{*}$.

(d) If $\beta \geq 2(1-\log 2) \approx 0.613706$, then $f \in \mathcal{S}_{e}^{*}$.

(e) If $-1<B<A<1$ and $\beta \geq \max \left\{\beta_{1}, \beta_{2}\right\}$ where

$$
\beta_{1}=\frac{2(1-\log 2)}{\log (1-B)-\log (1-A)} \text { and } \beta_{2}=\frac{2(\sqrt{2}-1+\log 2-\log (1+\sqrt{2}))}{\log (1+A)-\log (1+B)},
$$

then $f \in \mathcal{S}^{*}[A, B]$.

Next, we intend to determine a bound on $\beta$ so that $1+\beta z p^{\prime}(z) / p^{2}(z) \prec \sqrt{1+z}$ implies $p$ is subordinate to several well-known starlike functions.

Theorem 2.8. Let the function $p$ be analytic in $\mathbb{D}, p(0)=1$ and

$$
1+\beta z p^{\prime}(z) / p^{2}(z) \prec \sqrt{1+z} .
$$

Then the following subordination results hold for sharp bound of $\beta$ :

(a) If $\beta \geq 4(1+\sqrt{2})(1-\log 2) \approx 2.96323$, then $p(z) \prec \varphi_{0}(z)$. 
(b) If $\beta \geq \frac{2(1+\sin (1))(\sqrt{2}-\log (1+\sqrt{2})+\log 2-1)}{\sin (1)} \approx 0.989098$, then $p(z) \prec \varphi_{s}(z)$.

(c) If $\beta \geq(2+\sqrt{2})(\sqrt{2}-\log (1+\sqrt{2})+\log 2-1) \approx 0.771568$, then $p(z) \prec \varphi_{q}(z)$.

(d) Let $-1<B<A<1$ and $A_{0}=\frac{2-\log 4-\sqrt{2}+\log (1+\sqrt{2})}{\sqrt{2}-\log (1+\sqrt{2}+1)} \approx 0.151764$. If either

(i) $A>A_{0}$ and $\beta \geq \frac{2(1-A)(1-\log 2)}{A-B} \approx 0.613706 \frac{1-A}{A-B}$ or

(ii) $A<A_{0}$ and $\beta \geq \frac{2(1+A)(\sqrt{2}-1+\log 2-\log (1+\sqrt{2}))}{A-B} \approx 0.451974 \frac{1+A}{A-B}$, then $p(z) \prec(1+A z) /(1+B z)$.

Proof. The function $q_{\beta}: \overline{\mathbb{D}} \rightarrow \mathbb{C}$ defined by

$$
q_{\beta}(z)=\left(1-\frac{2}{\beta}(\sqrt{1+z}-\log (1+\sqrt{1+z})+\log 2-1)\right)^{-1}
$$

is clearly analytic and is a solution of the differential equation

$$
1+\beta z q_{\beta}^{\prime}(z) / q_{\beta}^{2}(z)=\sqrt{1+z} .
$$

Define the functions $\nu(w)=1$ and $\psi(w)=\beta / w^{2}$. The function $Q: \overline{\mathbb{D}} \rightarrow \mathbb{C}$ defined by

$$
Q(z)=z q_{\beta}^{\prime}(z) \psi\left(q_{\beta}(z)\right)=\beta z q_{\beta}^{\prime}(z) / q_{\beta}^{2}(z)=\sqrt{1+z}-1
$$

is starlike in $\mathbb{D}, Q$ is starlike function.

The function $h(z):=\nu\left(q_{\beta}(z)\right)+Q(z)=\nu\left(q_{\beta}(z)\right)+Q(z)$ satisfies the inequality $\operatorname{Re}\left(z h^{\prime}(z) / Q(z)\right)>0$ for $z \in \mathbb{D}$. Therefore, by using Lemma 2.2, we see that the subordination

$$
1+\beta \frac{z p^{\prime}(z)}{p^{2}(z)} \prec 1+\beta \frac{z q_{\beta}^{\prime}(z)}{q_{\beta}^{2}(z)}
$$

implies $p(z) \prec q_{\beta}(z)$. As the similar lines of the proof of Theorem 2.1, the proofs of parts (a)-(d) are obtained.

Remark 2.9. The subordination in part (d) of Theorem 2.8 was earlier investigated in $[9$, Lemma 2.4 , p. 6] where non-sharp result was obtained.

Remark 2.10. Let the function $f \in \mathcal{A}$ satisfies the following subordination

$$
1+\beta\left(\frac{z f^{\prime}(z)}{f(z)}\right)^{-1}\left(1-\frac{z f^{\prime}(z)}{f(z)}+\frac{z f^{\prime \prime}(z)}{f^{\prime}(z)}\right) \prec \sqrt{1+z} .
$$

Then the following are sufficient conditions for $f$ to be in various subclasses of $\mathcal{S}$.

(a) If $\beta \geq 4(1+\sqrt{2})(1-\log 2) \approx 2.96323$, then $f \in \mathcal{S}_{R}^{*}$.

(b) If $\beta \geq \frac{2(1+\sin (1))(\sqrt{2}-\log (1+\sqrt{2})+\log 2-1)}{\sin (1)} \approx 0.989098$, then $f \in \mathcal{S}_{s}^{*}$.

(c) If $\beta \geq(2+\sqrt{2})(\sqrt{2}-\log (1+\sqrt{2})+\log 2-1) \approx 0.771568$, then $f \in \mathcal{S}_{q}^{*}$.

(d) Let $-1<B<A<1$ and $A_{0}=\frac{2-\log 4-\sqrt{2}+\log (1+\sqrt{2})}{\sqrt{2}-\log (1+\sqrt{2}+1)} \approx 0.151764$.

If either $A>A_{0}$ and $\beta \geq \frac{2(1-A)(1-\log 2)}{A-B} \approx 0.613706 \frac{1-A}{A-B}$ or $A<A_{0}$ and

$$
\beta \geq \frac{2(1+A)(\sqrt{2}-1+\log 2-\log (1+\sqrt{2}))}{A-B} \approx 0.451974 \frac{1+A}{A-B},
$$

then $f \in \mathcal{S}^{*}[A, B]$. 


\section{References}

[1] Ali, R.M., Cho, N.E., Ravichandran, V., Kumar, S.S., Differential subordination for functions associated with the lemniscate of Bernoulli, Taiwanese J. Math., 16(2012), no. 3, 1017-1026.

[2] Ali, R.M., Ravichandran, V., Seenivasagan, N., Sufficient conditions for Janowski starlikeness, Int. J. Math. Math. Sci., 2007(2007), Art. ID 62925, 7 pp.

[3] Bulboacă, T., Differential Subordinations and Superordinations, Recent Results, House of Scientific Book Publ., Cluj-Napoca, 2005.

[4] Cho, N.E., Kumar, V., Kumar, S.S., Ravichandran, V., Radius problem for sin-starlike functions, Bull. Iranian Math. Soc. (to appear).

[5] Cho, N.E., Lee, H.J., Park, J.H., Srivastava, R., Some applications of the first-order differential subordinations, Filomat, 30(2016) no. 6, 1465-1474.

[6] Dorca, I., Breaz, D., Subordination of certain subclass of convex function, Stud. Univ. Babeş-Bolyai Math., 57(2012), no. 2, 181-187.

[7] Goluzin, G.M., On the majorization principle in function theory, Dokl. Akad. Nauk. SSSR, 42(1935), 647-650.

[8] Janowski, W., Extremal problems for a family of functions with positive real part and for some related families, Ann. Polon. Math., 23(1970/1971), 159-177.

[9] Kumar, S.S., Kumar, V., Ravichandran, V., Cho, N.E., Sufficient conditions for starlike functions associated with the lemniscate of Bernoulli, J. Inequal. Appl., 2013(2013), 176, $13 \mathrm{pp}$.

[10] Kumar, S., Ravichandran, V., A subclass of starlike functions associated with a rational function, Southeast Asian Bull. Math., 40(2016) no. 2, 199-212.

[11] Kumar, S., Ravichandran, V., Subordinations for Functions with Positive Real Part, Complex Anal. Oper. Theory, (2017), doi:10.1007/s11785-017-0690-4.

[12] Ma, W.C., Minda, D., A unified treatment of some special classes of univalent functions, in: Proceedings of the Conference on Complex Analysis (Tianjin, 1992), 157-169, Conf. Proc. Lecture Notes Anal., I, Int. Press, Cambridge, MA.

[13] Mendiratta, R., Nagpal, S., Ravichandran, V., On a subclass of strongly starlike functions associated with exponential function, Bull. Malays. Math. Sci. Soc., 38(2015), no. 1, 365386.

[14] Miller, S.S., Mocanu, P.T., On some classes of first-order differential subordinations, Michigan Math. J., 32(1985), no. 2, 185-195.

[15] Miller, S.S., Mocanu, P.T., Differential Subordinations: Theory and Applications, Dekker, New York, 2000.

[16] Nunokawa, M., Obradović M., Owa, S., One criterion for univalency, Proc. Amer. Math. Soc., 106(1989), no. 4, 1035-1037.

[17] Omar, R., Halim, S.A., Differential subordination properties of Sokót-Stankiewicz starlike functions, Kyungpook Math. J., 53(2013), no. 3, 459-465.

[18] Padmanabhan, K.S., Parvatham, R., Some applications of differential subordination, Bull. Austral. Math. Soc., 32(1985), no. 3, 321-330.

[19] Raina, R.K., Sokół, J., On coefficient estimates for a certain class of starlike functions, Hacet. J. Math. Stat., 44 (2015), no. 6, 1427-1433.

[20] Ravichandran, V., Sharma, K., Sufficient conditions for starlikeness, J. Korean Math. Soc., 52(2015) no. 4, 727-749. 
[21] Shanmugam, T.N., Convolution and differential subordination, Internat. J. Math. Math. Sci., 12(1989), no. 2, 333-340.

[22] Sharma, K., Jain, N.K., Ravichandran, V., Starlike functions associated with a cardioid, Afr. Mat., 27 (2016), no. 5-6, 923-939.

[23] Sharma, K., Ravichandran, V., Applications of subordination theory to starlike functions, Bull. Iranian Math. Soc., 42(2016) no. 3, 761-777.

[24] Sokół, J., Stankiewicz, J., Radius of convexity of some subclasses of strongly starlike functions, Zeszyty Nauk. Politech. Rzeszowskiej Mat., 19(1996), 101-105.

[25] Tuneski, N., Bulboacă, T., Jolevska-Tunesk, B., Sharp results on linear combination of simple expressions of analytic functions, Hacet. J. Math. Stat., 45(2016), no. 1, 121-128.

Om P. Ahuja

Kent State University

Department of Mathematics

Burton, USA

e-mail: oahuja@kent.edu

Sushil Kumar

Bharati Vidyapeeth's College of Engineering

A-4, Paschim Vihar

Delhi-110063, India

e-mail: sushilkumar16n@gmail.com

V Ravichandran

National Institute of Technology

Department of Mathematics

Tiruchirappalli - 620015, India

e-mail: vravi@maths.du.ac.in; vravi68@gmail.com 